\title{
Neonatal sepsis due to non-albicans Candida species and their susceptibility to antifungal agents: first report from Bangladesh
}

\author{
Rafia Afreen Jalii ${ }^{1^{*}}$, K.M. Shahidul Islam ${ }^{2}$, Lovely Barai ${ }^{2}$, Shahida Akhter ${ }^{3}$ \\ ${ }^{1}$ Department of Microbiology, Green Life Medical College, Green Road, Dhaka, Bangladesh, \\ ${ }^{2}$ Department of Microbiology, BIRDEM General Hospital, Shahbag, Dhaka, Bangladesh, \\ ${ }^{3}$ Department of Neonatology, BIRDEM General Hospital-2, Segunbagicha, Dhaka, Bangladesh
}

\begin{abstract}
Background and objectives: Frequency of neonatal sepsis in Neonatal Intensive Care Units (NICU) has been increasing worldwide over the last decades. The emergence of non-albicans Candida (NAC) species and their resistance to common antifungal agents become an important preventive and therapeutic issue. The present study was undertaken to find out the role of NAC species in neonatal sepsis/candidemia in the NICUs of hospitals of Dhaka city. The susceptibility pattern of NAC species to antifungal agents was also determined.
\end{abstract}

Materials and methods: Suspected cases of neonatal sepsis admitted in NICU of four tertiary care hospitals of Dhaka city, from March to December 2018 were enrolled. In this cross sectional study, blood samples were collected from neonates with suspected sepsis for culture. Identification of Candida species was done by carbohydrate $(\mathrm{CHO})$ assimilation tests using swab auxanographic technique, $\mathrm{CHO}$ impregnated yeast nitrogen base plate method (YNB), microtiter plate based miniaturized method and by HiCrome ${ }^{\mathrm{TM}}$ Candida Differential Media. Susceptibility of the isolated Candida species to antifungal agents was determined by disk diffusion (DD) and by minimum inhibitory concentration (MIC) methods. MIC was determined by broth microdilution method using RPMI 1640 and trypticase soy broth (TSB).

Results: In the present study, NAC species were isolated from $39.7 \%$ neonates. $C$. tropicalis was the predominant species $(81.0 \%)$ followed by $C$. parapsilosis $(12.1 \%)$, C. auris $(5.2 \%)$ and $C$. dubliniensis (1.7\%). Isolated NAC species were $98.3 \%$ sensitive to voriconazole. Sensitivity to fluconazole, ketoconazole, itraconazole, and clotrimazole was 3.5\%, 15.5\%, $86.2 \%$ and $56.9 \%$ respectively by DD method. All the isolates (100\%) were sensitive to miconazole and nystatin. All the $C$. tropicalis, $C$. auris and $C$. dubliniensis were sensitive to amphotericin B and anidulafungin. One and four $C$. parapsilosis were found resistant to amphotericin $B$ and anidulafungin respectively. The MIC results obtained by using RPMI 1640 and TSB as growth medium were concordant suggesting that TSB media was a good alternative to expensive RPMI 1640.

Conclusion: The advent of NAC species merits attention as they are highly resistant to most of the azoles. Therefore, speciation of Candida in neonatal candidemia is essential to institute appropriate antifungal therapy.

IMC J Med Sci 2020; 14(2): 005. EPub date: 13 January 2021

\section{Introduction}

Over the last two decades, blood stream infection (BSI) by Candida species has become a significant issue in neonatal intensive care units (NICUs).
Candidemia is the third most common cause of late onset sepsis in neonates. It is responsible for 9-13\% of BSI in neonates and is associated with high crude and attributable mortality rates [1].

*Correspondence: Rafia Afreen Jalil, Department of Microbiology, Green Life Medical College, Green Road, Dhaka, Bangladesh.Email: rafiaafreen133@gmail.com 
Among the Candida species, $C$. albicans is the most commonly isolated organism. But recently nonalbicans Candida (NAC) species have emerged as potential pathogens, particularly $C$. tropicalis, $C$. parapsilosis, C. krusei, C. glabrata and C. auris [2-4]. Various factors such as broad spectrum antibiotics, indwelling devices, prematurity, low birth weight (LBW), total parenteral nutrition (TPN), artificial ventilation and gastrointestinal surgery contribute to the risk of fungal colonization and infection. Also, fungal colonization is associated with overcrowding in the NICU, inadequate nurse-topatient ratio and poor hygiene practices. Approximately $10 \%$ of the newborns are colonized during the first week of life and up to $64 \%$ of them get colonized by 4 weeks stay in hospital [5-6]. Candida species may spread via vertical transmission from the maternal flora or by horizontal transmission from the healthcare workers (HCW) hands [7-8].

Majority of the Candida species become resistant to the antifungal agents, mainly to triazole compounds, by the expression of efflux pumps that minimize drug accumulation, altering the structure or concentration of antifungal target proteins and modification of membrane sterol composition $[9,10]$. Some NAC species are intrinsically resistant to fluconazole and newer triazoles. Therefore, speciation and antifungal susceptibility of all the yeast isolates are essential. Owing to significant regional heterogeneity, local epidemiological data is crucial in the prevention and management of invasive candidiasis.

No study has yet been carried out in Bangladesh on the frequency and the types of NAC species responsible for sepsis in neonates admitted at the NICUs of different hospitals. The present study was undertaken to determine the NAC species and their antifungal susceptibility pattern causing neonatal sepsis in the NICUs of four tertiary care hospitals of Dhaka city.

\section{Materials and methods}

This cross sectional hospital based study was carried out in the Department of Microbiology, Bangladesh Institute of Research and Rehabilitation in Diabetes, Endocrine and Metabolic Disorders
(BIRDEM) in collaboration with Department of Neonatology of four tertiary care hospitals of Dhaka city. The study period was from March, 2018 to December, 2018. The study was approved by the Institutional Ethical Committee of each institution and written informed consents from patients' guardian were obtained prior to collection of samples.

\section{Study population and collection of blood samples:} Neonates admitted in respective NICUs with suspected septicemia were included in the study. About 1-2 $\mathrm{ml}$ of peripheral venous blood samples were collected aseptically from enrolled neonates. Immediately, $0.5-1 \mathrm{ml}$ of blood was inoculated in BacT/Alert PF plus bottle and remaining $0.5-1 \mathrm{ml}$ blood inoculated in the lytic blood culture tube. The specimens were transported immediately to microbiology laboratory of BIRDEM. Since Candida could be part of skin flora of neonates admitted in hospital, its isolation from blood culture might reflect contamination from skin flora. To rule out this contamination, a second blood sample was collected from the culture positive cases.

Candidemia was diagnosed by isolation of Candida species from at least two consecutive blood samples with clinical features of septicemia.

Isolation and identification of NAC species: Culture was performed using standard microbiological techniques [11,12]. Candida was identified by colony morphology, wet film and Gram stain. Species identification was done by germ tube test, carbohydrate ( $\mathrm{CHO}$ ) assimilation tests using swab auxanographic technique, carbohydrate impregnated yeast nitrogen base plate method and microtitre plate based miniaturized method, modified enrichment broth growth assay and HiCrome $^{\mathrm{TM}}$ Candida Differential Media [13-16]. Yeast nitrogen base, bromocresol purple and eleven types of carbohydrates were used in all the three methods of $\mathrm{CHO}$ assimilation tests. In swab auxanographic method, carbohydrate was incorporated in individual discs. In the mictotitre plate and $\mathrm{CHO}$ impregnated YNB plate methods, the carbohydrates were incorporated in the media. Growth in the media and turning the bromocresol purple to yellow indicated utilization of particular 
carbohydrate. $C$. auris was further confirmed by modified enrichment broth growth assay with salt yeast nitrogen base broth. Growth at $42^{\circ} \mathrm{C}$ and development of a yellow colour in the medium indicated C. auris. The Candida isolates were inoculated on HiCrome ${ }^{\mathrm{TM}}$ Candida Differential Media and incubated at $37^{\circ} \mathrm{C}$ for 24 hours and the species were identified by colour of the colonies as per manufacturer's instructions.

Antifungal susceptibility tests: Antifungal susceptibility test was performed by disk diffusion method using Mueller-Hinton agar supplemented with $2 \%$ glucose and $0.5 \mu \mathrm{g} / \mathrm{ml}$ methylene blue dye. Inhibition zones for fluconazole and voriconazole were interpreted according to validated CLSI (M44A) [17], while for other drugs the inhibition zones were adopted from published studies [18-20]. Broth microdilution was done to determine minimum inhibitory concentration (MIC) of fluconazole, amphotericin $B$ and anidulafungin as per NCCLs M27-A2 and EUCAST v 7.3.1 [21,22] using both RPMI 1640 and trypticase soy broth as growth medium. For determination of minimal fungicidal concentration (MFC), $2 \mu \mathrm{l}$ of broth was withdrawn from the optically clear MIC well of respective antifungal agent (concentrations above the MIC) and plated on Sabouraud dextrose agar plate and incubated at $35^{\circ} \mathrm{C}$ for $72 \mathrm{hrs}$. MFC was defined as the lowest drug concentration that yielded less than three colonies, a killing activity of 99\% [23].

\section{Results}

A total of 146 neonates with suspected sepsis were enrolled in the study. Out of 146 suspected cases of neonatal sepsis, 91 (62.3\%) yielded positive blood culture. NAC species was isolated from 58 (39.7\%) cases and remaining $33(22.6 \%)$ yielded growth of bacteria (Table-1). Detail rate of isolation of NAC species from different categories of study population is shown in Table-1. Rate of isolation of NAC species from term and preterm babies were $19.1 \%$ and $43.2 \%$ respectively. Fungal culture positivity among normal birth weight (NBW), low birth weight (LBW) and very low birth weight (VLBW) babies were 26.3\%, 46.8\% and 33.3\% respectively. Of the 58 NAC species isolated, 3 were from neonates with early-onset sepsis and the rest 55 were from cases with late-onset sepsis. The ratio of isolation of NAC species in neonates of diabetic and non diabetic mother was 2:1. Table-2 shows that $C$. tropicalis was the predominant species (81.0\%) followed by C. parapsilosis $(12.1 \%)$, C. auris (5.2\%) and C. dubliniensis (1.7\%).

Susceptibility of NAC species to fluconazole, ketoconazole, itraconazole, clotrimazole and nystatin by disc diffusion method is shown in Table3. Out of 58 NAC isolates, $96.6 \%, 84.5 \%, 13.8 \%$ and $43.1 \%$ were resistant to fluconazole, ketoconazole, itraconazole and clotrimazole respectively. Except one $C$. parapsilosis isolate, none was resistant to voriconazole. None of the isolated NAC species was resistant to miconazole and nystatin.

Table-1: Rate of isolation of NAC species and bacteria from different category of study population

\begin{tabular}{lcccc}
\hline $\begin{array}{l}\text { Category of study } \\
\text { population }\end{array}$ & $\begin{array}{c}\text { Number of } \\
\text { cases }\end{array}$ & $\begin{array}{c}\text { Bacteria } \\
\mathbf{n}(\%)\end{array}$ & $\begin{array}{c}\text { NAC species } \\
\mathbf{n}(\%)\end{array}$ & $\begin{array}{c}\text { Total } \\
\mathbf{n}(\%)\end{array}$ \\
\hline All cases & 146 & $33(22.6)$ & $58(39.7)$ & $91(62.3)$ \\
Male & 99 & $19(19.2)$ & $49(49.5)$ & $68(68.7)$ \\
Female & 47 & $14(29.8)$ & $9(19.2)$ & $23(48.9)$ \\
Term & 21 & $1(4.8)$ & $4(19.1)$ & $5(23.8)$ \\
Preterm & 125 & $32(25.6)$ & $54(43.2)$ & $86(68.8)$ \\
NBW & 19 & $1(5.3)$ & $5(26.3)$ & $6(31.6)$ \\
LBW & 79 & $12(15.2)$ & $37(46.8)$ & $49(62.0)$ \\
VLBW & 48 & $20(41.7)$ & $16(33.3)$ & $36(75.0)$ \\
\hline
\end{tabular}

Note: NBW - normal birth weight, LBW - low birth weight, VLBW - very low birth weight. 
Table-2: Types of NAC species isolated from study population $(n=58)$

\begin{tabular}{lc}
\hline Candida species & Number (\%) \\
\hline C. tropicalis & $47(81.0)$ \\
C. parapsilosis & $7(12.1)$ \\
C. auris & $3(5.2)$ \\
C. dubliniensis & $1(1.7)$ \\
\hline
\end{tabular}

Table-4 shows the resistance pattern of isolated NAC species to fluconazole, amphotericin B and anidulafungin by MIC method. Among the $47 \mathrm{C}$. tropicalis, 44 (93.6\%) were resistant to fluconazole. C. parapsilosis, C. auris and C. dubliniensis were $100 \%$ resistant to fluconazole by both disc diffusion and MIC methods. All the $C$. tropicalis, $C$. auris and C. dubliniensis were sensitive to amphotericin B and anidulafungin. Out of 7 C. parapsilosis, 1
(14.3\%) and 4 (57.1\%) were found resistant to amphotericin $B$ and anidulafungin respectively. The MIC results obtained by using RPMI 1640 and TSB as growth medium were concordant suggesting that TSB media could be a good alternative to expensive RPMI 1640.

Table- 5 shows the $\mathrm{MIC}_{50}$ and $\mathrm{MIC}_{90}$ of fluconazole, amphotericin $B$ and anidulafungin of isolated NAC species. $\mathrm{MIC}_{50}$ value of fluconazole for all the NAC isolates were in the resistant range. $\mathrm{MIC}_{50}$ of amphotericin B for $C$. tropicalis, C. parapsilosis, C. auris and $C$. dubliniensis were $0.5 \mu \mathrm{g} / \mathrm{ml}, 1 \mu \mathrm{g} / \mathrm{ml}, 1$ $\mu \mathrm{g} / \mathrm{ml}$ and $0.25 \mu \mathrm{g} / \mathrm{ml}$ respectively and these values were all within the sensitive range. Only $\mathrm{MIC}_{90}$ of amphotericin B for C. parapsilosis was $2 \mu \mathrm{g} / \mathrm{ml}$ which was in the resistant range. $\mathrm{MIC}_{50}$ of anidulafungin for $C$. parapsilosis was $4 \mu \mathrm{g} / \mathrm{ml}$ and was in the resistant range. Both $\mathrm{MIC}_{50}$ and $\mathrm{MIC}_{90}$ of

Table-3: Antifungal susceptibility pattern of the isolated NAC species by disk diffusion method

\begin{tabular}{|c|c|c|c|c|c|c|c|c|}
\hline \multirow[b]{2}{*}{ Organism } & \multirow[b]{2}{*}{ Number } & \multicolumn{7}{|c|}{ Resistant $\mathrm{n}(\%)$ to } \\
\hline & & $\begin{array}{l}\frac{0}{0} \\
\widetilde{N} \\
\overline{0} \\
\frac{\Xi}{4} \\
\end{array}$ & 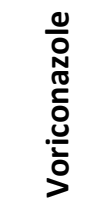 & 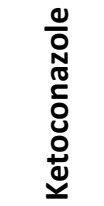 & 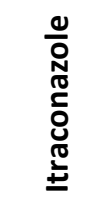 & 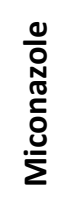 & 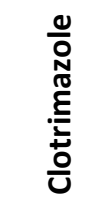 & 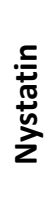 \\
\hline C. tropicalis & 47 & $\begin{array}{c}45 \\
(95.7)\end{array}$ & 0 & $\begin{array}{c}38 \\
(80.9)\end{array}$ & $\begin{array}{c}8 \\
(17.0)\end{array}$ & 0 & $\begin{array}{c}19 \\
(40.4)\end{array}$ & 0 \\
\hline C. parapsilosis & 7 & $\begin{array}{c}7 \\
(100)\end{array}$ & $\begin{array}{c}1 \\
(14.3)\end{array}$ & $\begin{array}{c}7 \\
(100)\end{array}$ & 0 & 0 & $\begin{array}{c}6 \\
(85.7)\end{array}$ & 0 \\
\hline C. auris & 3 & $\begin{array}{c}3 \\
(100)\end{array}$ & 0 & $\begin{array}{c}3 \\
(100)\end{array}$ & 0 & 0 & 0 & 0 \\
\hline C. dubliniensis & 1 & $\begin{array}{c}1 \\
(100)\end{array}$ & $\begin{array}{l}0 \\
0\end{array}$ & $\begin{array}{c}1 \\
(100)\end{array}$ & 0 & 0 & 0 & 0 \\
\hline Total & 58 & $\begin{array}{c}56 \\
(96.6)\end{array}$ & $\begin{array}{c}1 \\
(1.7)\end{array}$ & $\begin{array}{c}49 \\
(84.5)\end{array}$ & $\begin{array}{c}8 \\
(13.8)\end{array}$ & 0 & $\begin{array}{c}25 \\
(43.1)\end{array}$ & 0 \\
\hline
\end{tabular}

Table-4: Antifungal susceptibility pattern of isolated NAC species to fluconazole, amphotericin B and anidulafungin by MIC method

\begin{tabular}{lcccc}
\hline Candida sp. & Number & $\begin{array}{c}\text { Fluconazole } \\
\mathbf{n}(\%)\end{array}$ & $\begin{array}{c}\text { Amphotericin B } \\
\mathbf{n}(\%)\end{array}$ & $\begin{array}{c}\text { Anidulafungin } \\
\mathbf{n}(\%)\end{array}$ \\
\hline C. tropicalis & 47 & $44(93.6)$ & 0 & 0 \\
C. parapsilosis & 7 & $7(100)$ & $1(14.3)$ & $4(57.1)$ \\
C. auris & 3 & $3(100)$ & 0 & 0 \\
C. dubliniensis & 1 & $1(100)$ & 0 & 0 \\
\hline
\end{tabular}


Table-5: $M I C_{50}$ and $M I C_{90}$ of fluconazole, amphotericin $B$ and anidulafungin of isolated NAC species

\begin{tabular}{lccccccc}
\hline \multirow{2}{*}{ Candida sp } & \multirow{2}{*}{ Number } & \multicolumn{2}{c}{ Fluconazole } & \multicolumn{2}{c}{ Amphotericin $\mathbf{B}$} & \multicolumn{2}{c}{ Anidulafungin } \\
\cline { 3 - 8 } & & $\mathbf{M I C}_{\mathbf{5 0}}$ & $\mathbf{M I C}_{\mathbf{9 0}}$ & $\mathbf{M I C}_{\mathbf{5 0}}$ & $\mathbf{M I C}_{\mathbf{9 0}}$ & $\mathbf{M I C}_{\mathbf{5 0}}$ & $\mathbf{M I C}_{\mathbf{9 0}}$ \\
\hline C. tropicalis & 47 & 32 & 32 & 0.5 & 1 & 0.032 & 0.032 \\
C. parapsilosis & 7 & 16 & 32 & 1 & 2 & 4 & 4 \\
C. auris & 3 & 32 & 32 & 1 & 1 & 0.032 & 0.032 \\
C. dubliniensis & 1 & 16 & 16 & 0.25 & 0.25 & 0.008 & 0.008 \\
\hline
\end{tabular}

anidulafungin for rest of the Candida species were within the sensitive range. The lowest drug concentration required to inhibit the growth of $50 \%$ of organisms $\left(\mathrm{MIC}_{50}\right)$ and minimum concentration required to kill $50 \%$ of viable organisms $\left(\mathrm{MFC}_{50}\right.$ ) of the antifungal drugs for all the Candida species were calculated and shown in Table-6. MFC 50 was 2 fold higher than $\mathrm{MIC}_{50}$ for fluconazole, amphotericin $B$ and anidulafungin for all the NAC species.

Table-6: $M I C_{50}$ versus $M F C_{50}$ of fluconazole, amphotericin $B$ and anidulafungin for all NAC species

\begin{tabular}{lcc}
\hline $\begin{array}{l}\text { Antifungal } \\
\text { agents }\end{array}$ & $\begin{array}{c}\mathrm{MIC}_{50} \\
\mu \mathrm{g} / \mathrm{ml}\end{array}$ & $\begin{array}{c}\mathrm{MFC}_{50} \\
\boldsymbol{\mu g} / \mathrm{ml}\end{array}$ \\
\hline Fluconazole & 32 & 64 \\
Amphotericin B & 0.5 & 1 \\
Anidulafungin & 0.032 & 0.064 \\
\hline
\end{tabular}

\section{Discussion}

The present study has for the first time demonstrated the distribution of different nonalbicans Candida species responsible for sepsis among neonates admitted in the NICUs of different hospitals in Dhaka city. In the current study $39.7 \%$ neonates were culture positive for NAC species. ARTEMIS Antifungal Surveillance study conducted between June 1997 and December 2007 in 41 countries reported a declining trend in isolation of C. albicans from $70.9 \%$ to $65.9 \%$ [24]. A similar trend of emergence of NAC species in bloodstream infection has also been documented in a number of studies [25-31].

In this study, prematurity, LBW, VLBW, antibiotic prophylaxis and extended hospital stay could be the important reasons for high isolation of NAC species. Long-term use of broad-spectrum antibiotics in routine empiric therapy also contributes to an overgrowth of opportunistic Candida by reducing the competitive pressure imparted by normal bacterial flora [32]. Also, increased use of azole antifungal agents, particularly fluconazole, leads to an increase in the distribution of NAC species and a decrease in C. albicans [33].

In our study, we also observed higher rate of fungal septicemia in neonates born to diabetic mothers than that of non-diabetic mothers $(66.7 \%$ versus $22.5 \%$; $\mathrm{p}<0.05)$ ). Pregnant women with diabetes are at high risk of infection with Candida because the raised sugar promotes overgrowth of Candida and thus the neonates of the diabetic mothers are prone to be colonized with Candida from birth [34].

In the present study, among NAC species, $C$. tropicalis was the most common species (81.0\%). Various other studies have also reported $C$. tropicalis to be the most common isolate $[3,30,31]$. Pressure of fluconazole prophylaxis could be the reason behind this high rate of isolation of $C$. tropicalis in this study.

The isolated NAC species were $98.3 \%$ sensitive to voriconazole and this finding is similar to the results published by other studies $[35,36]$. In the present study, resistant rate to fluconazole by the Candida isolates was $87.9 \%$ by disk diffusion and $94.8 \%$ by MIC method. All the C. parapsilosis and C. auris were resistant to fluconazole while $C$. tropicalis isolates were $93.6 \%$ resistant. These findings were in agreement with Pandita et al. and Yadav et al $[37,38]$. All the $C$. tropicalis, $C$. auris and $C$. dubliniensis were sensitive to amphotericin $B$ and anidulafungin. Out of 7, only 1 (14.3\%) C. parapsilosis was found resistant to amphotericin B and 4 (57.1\%) were resistant to anidulafungin. Therefore, it appeared that amphotericin B and anidulafungin could be used against NAC species when the organisms become 
resistant to other antifungal agents. There are only few literatures available regarding the use of echinocandin particularly anidulafungin in the pediatric ICU. Anidulafungin might be used as an alternative drug in neonates particularly when the local Candida strains are resistant to azoles. However, since the first introduction of echinocandins, these antifungal agents have exhibited higher minimum inhibitory concentrations against $C$. parapsilosis. Compared to other species of Candida, C. parapsilosis demonstrates higher in vitro MICs to echinocandin, and treatment failures with these antifungal agents have been reported for $C$. parapsilosis infections $[39,40]$.

Antifungal susceptibility was done by disk-diffusion and MIC broth microdilution methods. RPMI 1640 is the proposed medium for carrying out micro broth dilution by EUCAST and NCCLs (M27-A2). However, RPMI 1640 is very expensive and not easily available in many laboratories. So, MIC of fluconazole and amphotericin B for Candida species were done using both TSB and RPMI 1640 medium and the results were compared. The MIC results obtained by both the media were concordant suggesting that TSB broth media could be a good alternative to RPMI 1640.

In our study, we observed the minimum fungicidal concentrations (MFC) of fluconazole, amphotericin $\mathrm{B}$ and anidulafungin as 2 fold higher than the MIC. Very little is known regarding the role of differences between MIC and MFC in treatment failure, and further studies are required.

The present study provided evidence of colossal burden of NAC species as an important cause of neonatal sepsis in our NICUs. The isolated NAC species were found highly resistant to widely used fluconazole whereas amphotericin B, voriconazole and anidulafungin were the most effective agents. The results of our study could be used as a template for the establishment of local guidelines for the effective treatment and prevention of neonatal candidemia.

\section{Competing interest}

The authors declared no competing interests.

\section{Funding}

None

\section{References}

1. Benjamin DK, Stoll BJ, Fanaroff AA, McDonald SA, Oh W, Higgins RD, et al. Neonatal candidiasis among extremely low birth weight infants: risk factors, mortality rates, and neurodevelopmental outcomes at 18 to 22 months. Pediatrics. 2006; 117(1): 84-92.

2. Oberoi JK, Wattal C, Goel N, Raveendran R, Datta S, Prasad K. Non-albicans Candida species in blood stream infections in a tertiary care hospital at New Delhi, India. Indian J Med Res. 2012; 136(6): 997-1003.

3. Goel N, Ranjan PK, Aggarwal R, Chaudhary U, Sanjeev N. Emergence of non-albicans Candida in neonatal septicemia and antifungal susceptibility: experience from a tertiary care center. J Lab Physicians. 2009; 1(2): 53-55.

4. Warris A. Candida auris, what do paediatricians need to know? Arch Dis Child. 2018; 103(9): 891-894.

5. Singhi S, Rao DS, Chakrabarti A. Candida colonization and candidemia in a pediatric intensive care unit. Pediatr Crit Care Med. 2008; 9(1): 91-95.

6. Rao $S$, Ali U. Systemic fungal infections in neonates. J Postgrad Med. 2005; 51 Suppl 1: S27-29.

7. Ariff S, Saleem AF, Soofi SB, Sajjad R. Clinical spectrum and outcomes of neonatal candidiasis in a tertiary care hospital in Karachi, Pakistan. $J$ Infect Dev Ctries. 2011; 5(03): 216-223.

8. Adib SM, Bared EE, Fanous R, Kyriacos S. Practices of Lebanese gynecologists regarding treatment of recurrent vulvovaginal candidiasis. N Am Med Sci. 2011; 3(9): 406-410.

9. Sanglard D, Odds FC. Resistance of Candida species to antifungal agents: molecular mechanisms and clinical consequences. Lancet Infect Dis. 2002; 2(2): 73-85.

10. Magill SS, Shields C, Sears CL, Choti M, Merz WG. Triazole cross-resistance among Candida spp.: case report, occurrence among bloodstream isolates, and implications for antifungal therapy. J Clin Microbiol. 2006; 44(2): 529-535. 
11. Cheesbrough M. District laboratory practice in tropical countries, part 2. Cambridge University Press; 2005.

12. Tarrand JJ, Guillot C, Wenglar M, Jackson J, Lajeunesse JD, Rolston KV. Clinical comparison of the resin-containing BACTEC 26 Plus and the Isolator 10 blood culturing systems. J Clin Microbiol. 1991; 29(10): 2245-2249.

13. McGinnis MR. Laboratory handbook of medical mycology. Michigan: Michigan University Press; 1980. p. 661.

14. Land GA, Vinton EC, Adcock GB, Hopkins JM. Improved auxanographic method for yeast assimilations: a comparison with other approaches. J Clin Microbiol. 1975; 2(3): 206-217.

15. Devadas SM, Ballal M, Prakash PY, Hande MH, Bhat GV, Mohandas V. Auxanographic carbohydrate assimilation method for large scale yeast identification. J Clin Diagn Res. 2017; 11(4): DC01-DC03.

16. Welsh RM, Bentz ML, Shams A, Houston $H$, Lyons $A$, Rose $L$, et al. Survival, persistence, and isolation of the emerging multidrugresistant pathogenic yeast Candida auris on a plastic health care surface. J Clin Microbiol. 2017; 55(10): 2996-3005.

17. Clinical and Laboratory Standards Institute. Methods for antifungal disk diffusion susceptibility testing of yeasts; Approved Guideline M44-A, Vol. 24, Number 15. 2nd ed. Wayne, USA: Clinical and Laboratory Standards Institute; 2004.

18. Pam VK, Akpan JU, Oduyebo OO, Nwaokorie FO, Fowora MA, Oladele RO, et al. Fluconazole susceptibility and ERG11 gene expression in vaginal Candida species isolated from Lagos Nigeria. Int J Mol Epidemiol Genet. 2012; 3(1): 84-90.

19. Dota KF, Freitas AR, Consolaro ME, Svidzinski TI. A challenge for clinical laboratories: detection of antifungal resistance in Candida species causing vulvovaginal candidiasis. $L a b$ Med. 2011; 42(2): 87-93.

20. Vandeputte $P$, Larcher $G$, Bergès $T$, Renier $G$, Chabasse D, Bouchara JP. Mechanisms of azole resistance in a clinical isolate of Candida tropicalis. Antimicrob Agents Chemother. 2005; 49(11): 4608-4615.

21. Clinical and Laboratory Standards Institute. Reference method for broth dilution antifungal susceptibility testing of yeasts M27-A2, Vol. 22, Number15. 2nd ed. Wayne, USA: Clinical and Laboratory Standards Institute; 2002.

22. European Society of Clinical Microbiology and Infectious Diseases. Method for the determination of broth dilution minimum inhibitory concentrations of antifungal agents for yeasts. EUCAST Definitive Document E.DEF 7.3. Copenhagen: European Society of Clinical Microbiology and Infectious Diseases; 2015.

23. Torres-Rodríguez JM, Alvarado-Ramírez E, Murciano F, Sellart M. MICs and minimum fungicidal concentrations of posaconazole, voriconazole and fluconazole for Cryptococcus neoformans and Cryptococcus gattii. J. Antimicrob Chemother. 2008; 62(1): 205-206.

24. Pfaller MA, Diekema DJ, Gibbs DL, Newell VA, Ellis $D$, Tullio $V$, et al. Results from the ARTEMIS DISK Global Antifungal Surveillance Study, 1997 to 2007: a 10.5-year analysis of susceptibilities of Candida species to fluconazole and voriconazole as determined by CLSI standardized disk diffusion. J Clin Microbiol. 2010; 48(4): 1366-1377.

25. Kossoff EH, Buescher ES, Karlowicz MG. Candidemia in a neonatal intensive care unit: trends during fifteen years and clinical features of 111 cases. Pediatr Infect Dis J. 1998; 17(6): 504-508.

26. Sardana V, Pandey A, Madan M, Goel SP, Asthana AK. Neonatal candidemia: a changing trend. Indian J Pathol Microbiol. 2012; 55(1): 132-133.

27. Rani R, Mohapatra NP, Mehta G, Randhawa VS. Changing trends of Candida species in neonatal septicaemia in a tertiary North Indian hospital. Indian J Med Microbiol. 2002; 20(1): 42-44.

28. Femitha $P$, Joy $R$, Adhisivam $B$, Bhat $V$, Prasad $K$, Gane BD, Singh R. Candidemia in neonatal ICU-experience from a tertiary care hospital. Skin. 2013; 5: 13-19.

29. Shrivastava G, Bajpai T, Bhatambare GS, Chitnis $V$, Deshmukh AB. Neonatal candidemia: Clinical 
importance of species identification. Sifa Med J. 2015; 2(2): 37.

30. Capoor MR, Nair D, Deb M, Verma PK, Srivastava L, Aggarwal P. Emergence of nonalbicans Candida species and antifungal resistance in a tertiary care hospital. Jpn J Infect Dis. 2005; 58(6): 344-348.

31. Agarwal J, Bansal S, Malik GK, Jain A. Trends in neonatal septicemia: emergence of nonalbicans Candida. Indian pediatrics. 2004 41(7): 712-715.

32. Benjamin DK, Stoll BJ, Gantz MG, Walsh MC, Sánchez PJ, Das A, et al. Neonatal candidiasis: epidemiology, risk factors, and clinical judgment. Pediatrics. 2010; 126(4): e865-e873.

33. Filioti J, Spiroglou K, Roilides E. Invasive candidiasis in pediatric intensive care patients: epidemiology, risk factors, management, and outcome. Intensive Care Med. 2007; 33(7): 1272-1283.

34. Sopian IL, Sa'adiah Shahabudin MA, Lung LT, Sandai D. Yeast infection and diabetes mellitus among pregnant mother in Malaysia. Malays $J$ Med Sci. 2016; 23(1): 27-34.
35. Kavitha H, Anuradha K, Venkatesha D Comparison of susceptibility of various Candida species isolated from neonatal septicaemia to voriconazole and fluconazole. IOSR J Pharm Biol Sci. 2014; 9(02): 78-81.

36. Nazir A. Non-albicans Candida in neonatal septicemia: an emerging clinical entity. Int $J$ Biomed Res. 2016; 7(2): 47-50.

37. Pandita N, Peshin C, Wasim S, Bhat NK, Gupta A. Profile of fungal septicaemia in new born at a tertiary care hospital in North India. Int J Contemp Pediatr. 2017; 4(2): 455-459.

38. Yadav S, Dahiya S, Budhani D. Candidemia in neonatal intensive care unit: a cause of concern. Int J Res Med Sci. 2017; 5(5): 21652167.

39. Arendrup $M C$, Perlin DS. Echinocandin resistance: an emerging clinical problem? Curr Opin Infect Dis. 2014; 27(6): 484-492.

40. Perlin DS. Echinocandin resistance, susceptibility testing and prophylaxis: implications for patient management. Drugs. 2014; 74(14): 1573-1585. 